\title{
Prognostic relevance of $K R A S$ genotype in metastatic colorectal cancer patients unfit for FIr-B/FOx intensive regimen
}

\author{
GEMMA BRUERA $^{1,4}$, KATIA CANNITA ${ }^{1}$, ALDO VICTOR GIORDANO ${ }^{2}$, ROBERTO VICENTINI ${ }^{3}$, \\ CORRADO FICORELLA ${ }^{1,4}$ and ENRICO RICEVUTO ${ }^{1,4}$ \\ ${ }^{1}$ Medical Oncology, ${ }^{2}$ Radiology, ${ }^{3}$ Hepatobiliar-Pancreatic Surgery, S. Salvatore Hospital, \\ ${ }^{4}$ Department of Biotechnological and Applied Clinical Sciences, \\ University of L'Aquila, I-67100 L'Aquila, Italy
}

Received December 13, 2013; Accepted February 7, 2014

DOI: $10.3892 /$ ijo.2014.2369

\begin{abstract}
First-line triplet chemotherapy plus bevacizumab (FIr-B/FOx) can improve efficacy of metastatic colorectal cancer (MCRC), KRAS wild-type and mutant. Prognostic relevance of $K R A S$ genotype was evaluated in patients unfit for FIr-B/FOx, treated with conventional medical treatments. Consecutive MCRC patients not eligible for FIr-B/FOx regimen due to age ( $\geq 75$ years) and/or comorbidities were treated with tailored conventional first-line treatments. KRAS codon 12/13 mutations were screened by direct sequencing. Activity and efficacy were evaluated and compared according to medical treatments, age (non-elderly and elderly $\geq 65$ years), comorbidity stage (Cumulative Illness Rating Scale), metastatic extension (liver-limited and other/multiple metastatic), and $K R A S$ genotype, using log-rank. Selected first line treatments were medical in 37 patients (92.5\%), and surgical in 3 patients (7.5\%). Medical treatment regimens: triplet, 18 (45\%); doublet, 15 (37.5\%); mono-therapy, 4 (10\%). At median follow-up of 8 months, objective response rate (ORR) was $37 \%$, median progression-free survival (PFS) 7 months, liver metastasectomies $8 \%$ (liver-limited disease $37.5 \%$ ), median overall survival (OS) 13 months. Triplet regimens failed to significantly affect clinical outcome, compared to doublet. According to KRAS genotype, ORR, PFS and OS were, respectively: wild-type 50\%, 8 months, 13 months; mutant 25\%, 6 months, 9 months. $K R A S$ genotype wild-type compared to mutant significantly affected PFS, while not OS. KRAS c.35 G>A mutation (G12D) significantly affected worse PFS and OS compared to wildtype and/or other mutations. KRAS genotype, specifically the
\end{abstract}

Correspondence to: Professor Enrico Ricevuto, Department of Biotechnological and Applied Clinical Sciences, U.O.C. Medical Oncology, S. Salvatore Hospital, University of L'Aquila, Via Vetoio, Coppito (L'Aquila), I-67100, Italy

E-mail: enrico.ricevuto@univaq.it

Key words: c.35 G>A KRAS mutation, elderly, FIr-B/FOx, KRAS genotype, metastatic colorectal cancer, triplet chemotherapy plus bevacizumab c.35 G>A KRAS mutation, may indicate poor prognosis in MCRC patients unfit for intensive medical treatments.

\section{Introduction}

Clinical management of metastatic colorectal cancer (MCRC) faces with different options and lines of treatment according to patients' fitness [age, performance status (PS), comorbidities], extension of metastatic disease [liver-limited (L-L) or other/ multiple metastatic (O/MM)], KRAS genotype (1-4). First line triplet chemotherapy, or doublets plus bevacizumab (BEV) or cetuximab, reported overlapping activity and efficacy in phase III trials, ranging between ORR 39-68\%, PFS 7.2-10.6 months and OS 19.9-26.1 months $(2,5,6)$. More intensive regimens, consisting of triplet chemotherapy plus targeted agents, can further increase activity, efficacy and effectiveness of liver metastasectomies (7-9).

In clinical practice, a decision-making process including functional, nutritional, and co-morbidity status is required to tailor first line medical treatment (10). Elderly status (age $>65$ years), PS >2, and/or comorbidities represent major features, to limit toxicities and maintain quality of life (QoL). Elderly MCRC patients are prevalent, and a clinical challenge is to select between intensive or tailored medical treatments, by properly weighing expected safety and efficacy, and according to prognostic factors. Retrospective studies showed that elderly patients benefit from 5-fluorouracil (5-FU) (11-13), irinotecan (CPT-11)-containing therapy $(14,15)$, FOLFOX (16) to the same extent as younger (17-19). In the OPTIMOX1 trial, ORR 59\%, PFS 9.0 months and OS 20.7 months were comparable between old-elderly and younger patients treated with FOLFOX (20). Treatment efficacy was also comparable with BEV associated to 5-FU/CPT-11 (21). In elderly patients, addition of BEV to 5-FU based chemotherapy significantly prolonged PFS (9.2-9.3 months) and OS (17.4-19.3 months) $(22,23)$. In BRiTE and BEAT studies, no different PFS was observed in elderly patients; median OS decreased with age $(24,25)$. In the randomized phase III trial comparing FOLFIRI with FOLFOXIRI, age was not significantly related to activity and efficacy, with OS 16.9 and 19.9 months, respectively $(26,27)$. ORR was significantly lower in older patients treated with FOLFOXIRI (27). Patients underwent metastasectomies 
without increased morbidity or mortality, irrespective of age. Patients with PS 2 presented a significantly lower OS and PFS, irrespectively of FOLFIRI or FOLFOXIRI chemotherapy regimen (27). Age and/or comorbidities did not affect efficacy in patients treated with cetuximab added to FOLFOX or FOLFIRI (28). In elderly and PS 2 patients, PFS was not increased by addition of panitumumab to FOLFOX (29). A meta-analysis showed that PS 1 compared to PS 2 significantly affect prognosis, regardless of treatment, with ORR 43.8 vs $32 \%$, PFS 7.6 vs 4.9 months, OS 17.3 and 8.5 months, respectively (30). The FOCUS2 randomized trial prospectively evaluated first line chemotherapy options consisting of $80 \%$ dose 5-FU or capecitabine, with or without oxaliplatin (OXP), in old-elderly and/or frail patients, and showed that addition of OXP significantly improved ORR (35 vs 13\%), a trend of PFS (5.8 vs. 4.5 months, hazard ratio $0.84, \mathrm{p}=0.07$ ), but not OS (31), without significantly increasing toxicity, with a negative impact on QoL.

KRAS mutations occur in $35-45 \%$ of colorectal cancer (CRC), mostly codon 12 (80\%), prevalently c.35 G>A (G12D) transversion $(32.5 \%)(32,33)$, impairing the intrinsic GTPase activity, and leading to constitutive, growth factor receptorindependent activation of downstream signalling (34). In the in vitro model proposed by Guerrero et al (35), codon 12 mutations increase aggressiveness by the differential regulation of KRAS downstream pathways that lead to inhibition of apoptosis, enhanced loss of contact inhibition and increased predisposition to anchorage-independent growth. KRAS genotype, wild-type or mutant, addresses the addition of targeted agents in MCRC medical treatment: anti-EGFR or anti-VEGF to doublet chemotherapy in KRAS wild-type (36-39); BEV to 5-FU, CPT-11 in KRAS mutant, significantly predicting prolonged PFS, while not OS and activity $(36,37)$.

Clinical outcome in wild-type and mutant patients assesses the prognostic relevance of $K R A S$ genotype, depending on differential tumor biological aggressiveness (4), including the predictive effectiveness of treatment strategies. Median OS of patients treated with BEV added to CPT-11/5-FU or triplet chemotherapy was different in $K R A S$ wild-type and mutant patients, but not significantly $(4,8,36,37)$; KRAS wild-type L-L patients may achieve a significantly greater benefit from integration with liver metastasectomies, with respect to mutant patients (4). We recently reported that the prevalent KRAS c.35 G>A (G12D) mutant genotype may significantly affect worse OS of MCRC patients treated with FIr-B/FOx, compared to wild-type or different other mutations (40). Here, we report a retrospective exploratory analysis evaluating tailored first line treatments, the prognostic value of KRAS genotype, and of the c.35 G>A mutation, in consecutive MCRC patients not eligible for intensive first line FIr-B/FOx expanded clinical program, due to age and/or comorbidities.

\section{Materials and methods}

Patient eligibility. Consecutive MCRC patients not eligible, due to comorbidities and/or age, for expanded clinical program or ongoing phase II trial proposing intensive regimens consisting of triplet chemotherapy plus targeted agent, were treated in clinical practice with first line medical and/ or surgical treatments, chosen among those in indication for MCRC treatment and approved by Agenzia Italiana del Farmaco (AIFA) for administration in label in Italian public hospitals, and published in Gazzetta Ufficiale Repubblica Italiana ('Elenco dei Medicinali erogabili a totale carico del Servizio sanitaria nazionale', Gazzetta Ufficiale Repubblica Italiana N.1, 2 Gennaio 2009). Thus, it was not a clinical trial and approval by ethics committee and institutional review board was not necessary, because patients were treated with conventional treatments without any additional medical intervention out of the best common clinical practice. Patients had histological confirmed diagnosis of MCRC, age $\geq 18$ years, PS $\leq 2$. Criteria to define patients unfit, or not eligible for intensive regimens were: age $\geq 75$ years; uncontrolled severe diseases; cardiovascular disease (uncontrolled hypertension, uncontrolled arrhythmia, ischemic cardiac diseases in the last year); thromboembolic disease, coagulopathy, preexisting bleeding diatheses; proteinuria $>1$ g/24 h. Patients were classified according to Cumulative Illness Rating Scale (CIRS) (10). Treatment options were tailored according to age ( $<$ or $\geq 75$ years), patient's fitness (PS, CIRS), KRAS genotype. Patients with PS 3 were not treated. All patients provided written, informed consent to the proposed in label treatment option.

\section{Methods}

Medical treatment regimens. Medical treatments included triplet, doublet, or mono-chemotherapy. Triplet FIr/FOx schedule consisted of weekly timed-flat-infusion 5-FU (TFI 5-FU), associated to weekly alternating CPT-11 or L-OXP (41): TFI/5-FU (Fluorouracil Teva; Teva Italia, Milan, Italy), $750-900 \mathrm{mg} / \mathrm{m}^{2} / \mathrm{die}$, over $12 \mathrm{~h}$ (from 10:00 pm to 10:00 am), days 1-2, 8-9, 15-16, 22-23; CPT-11 (Campto; Pfizer, Latina, Italy), 120-160 mg/m², days 1 and 15; 1-OXP (Eloxatin; SanofiAventis, Milan, Italy), $70-80 \mathrm{mg} / \mathrm{m}^{2}$, days 8 and 22; cycles every 4 weeks. Other triplet, doublet and mono-regimens were administered according to previously reported schedules $(7,41,42)$. Targeted agents were: BEV (Avastin; Roche, Welwyn Garden City, UK), 5 mg/kg, days 1 and 15; cetuximab (Erbitux; Merck, Darmstadt, Germany), $400 \mathrm{mg} / \mathrm{m}^{2}$ initial dose, then $250 \mathrm{mg} / \mathrm{m}^{2} /$ week.

Mutational analysis. Genetic analyses were performed on paraffin-embedded tissue blocks from primary tumor and/ or metastatic sites, as previously reported (4). Genotype status was assessed for KRAS codon 12 and 13 mutations by direct sequencing. KRAS exon 2 sequence was performed from PCR-amplified tumor DNA using the Big Dye V3.1 Terminator kit, electrophoresis in ABI PRISM 3130xl Genetic Analyzer, and analysis using the GeneMapper Analysis software version 4.0 (Applied Biosystems, Foster City, CA, USA).

Study design. Activity, efficacy, and prognostic relevance of first line treatments, and KRAS genotype on clinical outcomes were evaluated. Patients were classified according to: metastatic extension, L-L and O/MM (3,4); age, non-elderly ( $<65$ years), young-elderly ( $\geq 65<75$ years), old-elderly ( $\geq 75$ years); CIRS stage primary, intermediate, secondary. Clinical evaluation of response was made by CT scan; PET was added based on investigators' assessment. Follow-up was scheduled every two-three months up to disease progression or death. L-L patients were 
Table I. First line clinical management of unfit MCRC patients.

\begin{tabular}{|c|c|c|c|}
\hline & \multirow{2}{*}{$\frac{\text { Overall }}{\text { No. of patients }(\%)}$} & \multicolumn{2}{|c|}{$K R A S$ genotype } \\
\hline & & Wild-type (\%) & Mutant (\%) \\
\hline Total no. & 40 & 23 & 16 \\
\hline Medical treatment & $37(92.5)$ & $21(91)$ & $15(94)$ \\
\hline Triplet regimen & $18(45)$ & $8(35)$ & $10(62.5)$ \\
\hline Doublet chemotherapy plus bevacizumab & 3 & 1 & 2 \\
\hline Doublet chemotherapy plus cetuximab & 5 & 5 & - \\
\hline Triplet chemotherapy & 10 & 2 & 8 \\
\hline Doublet regimen & $15(37.5)$ & $12(52)$ & $3(19)$ \\
\hline Mono-chemotherapy plus bevacizumab & 2 & - & 2 \\
\hline Mono-chemotherapy plus cetuximab & 8 & 8 & - \\
\hline Doublet chemotherapy & 5 & 4 & 1 \\
\hline Mono-therapy & $4(10)$ & $1(4)$ & $2(12.5)$ \\
\hline Mono-chemotherapy & 4 & 1 & 2 \\
\hline Surgery & 3 & 2 & 1 \\
\hline
\end{tabular}

evaluated at baseline and every two-three cycles of treatment by a multidisciplinary team, to evaluate resectability defined according to reported categories (3). Liver metastasectomies were defined as $\mathrm{R} 0$, if radical surgery, $\mathrm{R} 1$, if radioablation was added. Surgery was recommended $>4$ weeks after BEV discontinuation.

Clinical criteria of activity and efficacy were ORR, resection rate of metastases, PFS and OS: ORR, evaluated according to RECIST criteria (43); pathologic complete response, defined as no residual cancer cells in surgical specimens; PFS and OS, evaluated using the Kaplan-Meier method (44). PFS was defined as the length of time from the beginning of treatment and disease progression or death (resulting from any cause) or to the last contact; OS as the length of time between the beginning of treatment and death or to last contact. Log-rank test was used to compare PFS and OS according to medical treatment, KRAS genotype, metastatic extension, age and comorbidity stage (45).

\section{Results}

Patient demographics. Forty patients unfit for intensive regimens, among 72 consecutive MCRC (56\%), were treated with (Table I): medical treatments, 37 patients $(92.5 \%)$; surgery, $3(7.5 \%)$. First line medical treatments: triplet, 18 (45\%); doublet, 15 (37.5\%); mono-therapy, 4 (10\%). Among 39 KRAS evaluated patients (97.5\%), $23(59 \%)$ were wild-type and $16(41 \%)$ mutant. Clinical features of the 37 patients who underwent first line medical treatments were (Table IIA): male/female ratio, 22/15; median age, 75 years; young- and old-elderly, $28(76 \%)$ and 20 (54\%), respectively; PS 0, 15 (41\%) and 1-2, 22 (59\%); metastatic disease metachronous $24 \%$, synchronous $76 \%$. Liver metastases, 26 patients (70\%); L-L 8 (22\%), O/MM 29 79\%). Distribution of patients according to age and comorbidity stage (Table IIB): non-elderly 9 (24\%), youngelderly $8(22 \%)$, old-elderly $20(54 \%)$; CIRS stage primary
$4(11 \%)$, intermediate 15 (40\%), secondary $18(42 \%)$. KRAS mutations detected in 15 patients were: codon $12,13(36.1 \%)$, specifically c.35 G>A (G12D), 7 (19.4\%), c.35 G>T (G12V), 6 (16.6\%); codon 13, 2 (5.5\%), c.37 G>T (G13V), 1 (2.7\%) and c. $38 \mathrm{G}>\mathrm{A}(\mathrm{G} 13 \mathrm{D}), 1(2.7 \%)$.

Medical treatments were tailored according to age and CIRS stage. Triplet regimens were administered in 18 patients (49\%): non-elderly 6, young-elderly 4, old-elderly 8; CIRS primary 2 , intermediate 10 , secondary 6 . Doublet regimens were administered in 15 patients $(40 \%)$ : non-elderly 3 , youngelderly 3, old-elderly 9; CIRS primary 1 , intermediate 4, secondary 10. Mono-regimens were administered in 4 patients $(11 \%)$ : young-elderly 1 , old-elderly 3 ; CIRS primary 1 , intermediate 1 , secondary 2 .

Overall activity and efficacy. Among the 37 patients who underwent medical treatments, 10 were not evaluable for activity: 7 (19\%) did not receive at least 2 cycles of treatment; 3 were on-treatment. The intent-to-treat analysis of 27 patients showed ORR 37\% ( $\alpha 0.05, \mathrm{CI} \pm 19)$ (Table IIIA). We observed 10 objective responses: 9 partial (33\%) and 1 complete (CR 4\%); 9 stable diseases (33\%); 8 progressive diseases (30\%). Disease control rate was $67 \%(\alpha 0.05, \mathrm{CI} \pm 18)$. After median follow-up of 8 months, median PFS was 7 months (1-13+): 28 events occurred. Median OS was 13 months (1+-23+): 22 events occurred (Fig. 1A). R0 liver metastasectomies were performed in 3 patients (8\%): 3 out of 8 L-L (37.5\%). No surgery-related complications were reported. Overall, 1 clinical plus 1 pathologic CR were reported (7\%); 1 patient showed a progressive disease at 8 months; 1 patient was progression-free at 10 months. Pathologic CR was obtained in $1 K R A S$ wild-type patient $(33 \%)$, with primary rectal tumor and a single $\mathrm{L}-\mathrm{L}$ metastasis. Twelve patients $(32 \%)$ received, at least, a second line treatment.

Among 7 evaluable L-L patients, ORR was $71 \%$; 3 performed liver metastasectomies (43\%) and $1 \mathrm{cCR}(14 \%)$; 
Table II. Patients' distribution according to features and age/comorbidity stage.

\begin{tabular}{|c|c|c|c|}
\hline \multicolumn{4}{|l|}{ A, Features of the unfit patients } \\
\hline & $\begin{array}{l}\text { Overall treated } \\
\text { Total no. }(\%)\end{array}$ & $\begin{array}{l}K R A S \text { wild-type } \\
\text { Total no. }(\%)\end{array}$ & $\begin{array}{l}\text { KRAS mutant } \\
\text { Total no. }(\%)\end{array}$ \\
\hline No. of patients & 37 & $21(58)$ & $15(42)$ \\
\hline \multicolumn{4}{|l|}{ Gender } \\
\hline Male/female & $22 / 15$ & $12 / 9$ & $10 / 5$ \\
\hline \multicolumn{4}{|l|}{ Age, years } \\
\hline Median & 75 & 77 & 69 \\
\hline Range & $45-87$ & $45-83$ & $50-87$ \\
\hline \multicolumn{4}{|l|}{ Elderly } \\
\hline$\geq 65$ years & $28(76)$ & $18(86)$ & $9(60)$ \\
\hline$\geq 75$ years & $20(54)$ & $13(62)$ & $7(47)$ \\
\hline \multicolumn{4}{|l|}{ WHO performance status } \\
\hline 0 & $15(41)$ & $10(48)$ & $5(33)$ \\
\hline $1-2$ & $22(59)$ & $11(52)$ & $10(69)$ \\
\hline \multicolumn{4}{|l|}{ CIRS stage } \\
\hline Primary & $4(11)$ & $1 \quad(5)$ & $2(13)$ \\
\hline Intermediate & $15(41)$ & $7(33)$ & $8(53)$ \\
\hline Secondary & $18(48)$ & $13(62)$ & $5(33)$ \\
\hline \multicolumn{4}{|l|}{ Metastatic disease } \\
\hline Metachronous & $9(24)$ & $5(24)$ & $3(20)$ \\
\hline Synchronous & $28(76)$ & $16(76)$ & $12(80)$ \\
\hline \multicolumn{4}{|l|}{ Primary tumor } \\
\hline Colon & $25(68)$ & $13(62)$ & $12(80)$ \\
\hline Rectum & $12(32)$ & $8(38)$ & $3(20)$ \\
\hline \multicolumn{4}{|l|}{ Sites of metastases } \\
\hline Liver & $26(70)$ & $16(76)$ & $9(60)$ \\
\hline Lung & $14(38)$ & $6(29)$ & $8(53)$ \\
\hline Lymph nodes & $11(30)$ & $6(29)$ & $4(27)$ \\
\hline Local & 7 (19) & $2(9)$ & $4(27)$ \\
\hline Other & $7(19)$ & $4(19)$ & $3(20)$ \\
\hline \multicolumn{4}{|l|}{ No. of involved sites } \\
\hline 1 & $14(38)$ & $9(43)$ & $5(33)$ \\
\hline$\geq 2$ & $23(62)$ & $12(57)$ & $10(69)$ \\
\hline \multicolumn{4}{|l|}{ Single metastatic sites } \\
\hline Liver-limited & $8(22)$ & $7(33)$ & $1 \quad(7)$ \\
\hline Other than liver & $8(22)$ & $3(14)$ & $5(33)$ \\
\hline Lung & $3(8)$ & - & $3(20)$ \\
\hline Lymph nodes & $1 \quad(3)$ & $1 \quad(5)$ & - \\
\hline Local & $4(11)$ & $2(9)$ & $2(13)$ \\
\hline Multiple metastatic sites & $21(57)$ & $11(52)$ & $9(60)$ \\
\hline \multicolumn{4}{|l|}{ Liver metastases } \\
\hline Single & 3 (8) & $3(14)$ & - \\
\hline Multiple & $23(62)$ & $13(62)$ & $9(60)$ \\
\hline Previous adjuvant chemotherapy: & 7 (19) & $2(9)$ & $5(33)$ \\
\hline FA/5-FU bolus & $1(3)$ & $1(5)$ & - \\
\hline XelOx or 5-FU/OXP & $6(16)$ & $1(5)$ & $5(33)$ \\
\hline Previous radiotherapy: & $4(11)$ & $3(14)$ & $1 \quad(7)$ \\
\hline RT+CT (5-FU continous infusion) & $3(8)$ & $2(9)$ & 1 (7) \\
\hline $\mathrm{RT}+\mathrm{CT}(\mathrm{XELOX})$ & $1 \quad(3)$ & $1 \quad(5)$ & - \\
\hline
\end{tabular}

WHO, World Health Organization; CIRS, Cumulative Illness Rating Scale. 
Table II. Continued.

B, Age and comorbidity stage in unfit patients

\begin{tabular}{lllcr}
\hline & \multicolumn{2}{c}{ Cumulative illness rating scale (CIRS) } \\
\cline { 2 - 4 } Age & Primary & Intermediate & Secondary & \multicolumn{1}{c}{$\begin{array}{c}\text { Total } \\
\text { no. }(\%)\end{array}$} \\
\hline Non-elderly & 2 & 5 & 2 & $9(24)$ \\
Young-elderly & 1 & 3 & 4 & $8(22)$ \\
Old-elderly & 1 & 7 & 12 & $20(54)$ \\
Total no. $(\%)$ & $4(11)$ & $15(40)$ & $18(49)$ & 37
\end{tabular}

A1

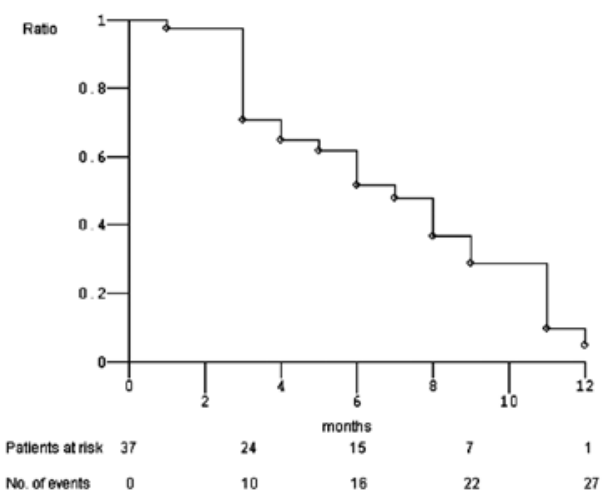

7 months (1-13+)

\section{B1}

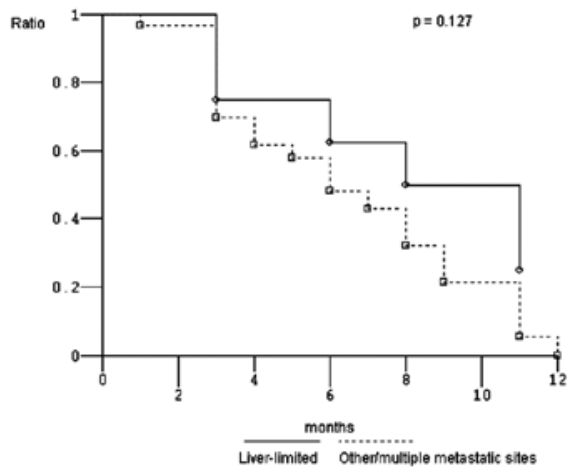

\section{C1}

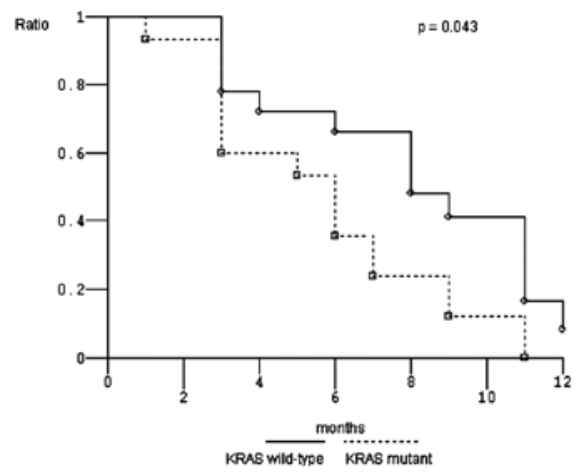

median PFS 11 months (3-13+ months); median OS 12 months (3-13+ months). Among 20 evaluable O/MM patients, ORR was 25\%; median PFS 6 months (1-12 months); median OS 13 months (1+-23+ months). Clinical outcome (PFS and OS) in $\mathrm{L}-\mathrm{L}$ compared to $\mathrm{O} / \mathrm{MM}$ patients was not significantly different (Fig. 1B).

Activity and efficacy according to first line treatment, elderly and comorbidity status. Among 16 evaluable patients treated with triplet regimens (Table IIIB), ORR was 37.5\% ( $\alpha 0.05$, $\mathrm{CI} \pm 24)$. We observed 6 partial responses (37.5\%); 5 stable diseases (31\%); 5 progressive diseases (31\%). Median PFS was

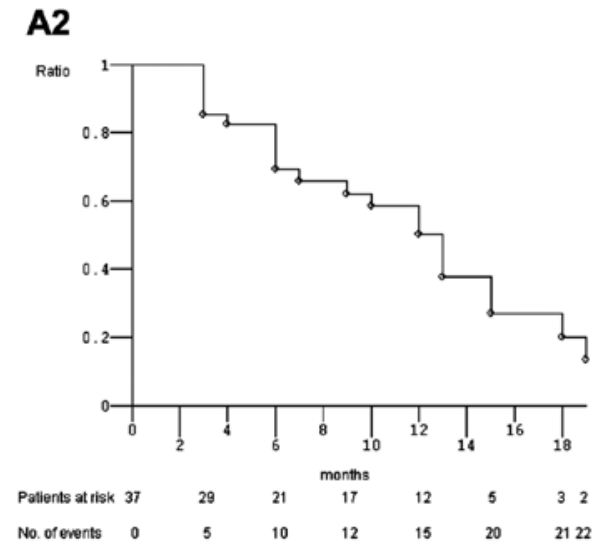

13 months $(1+-23+)$

B2

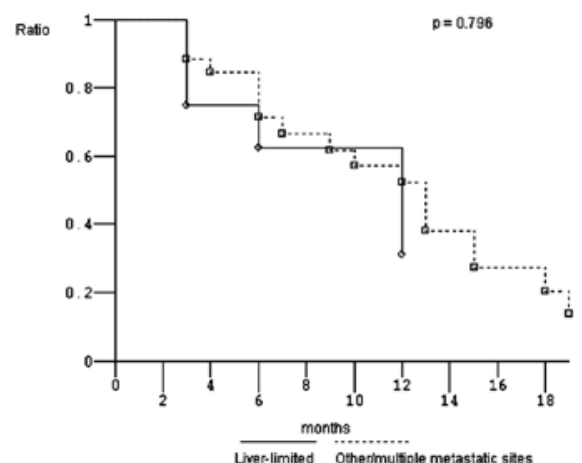

C2

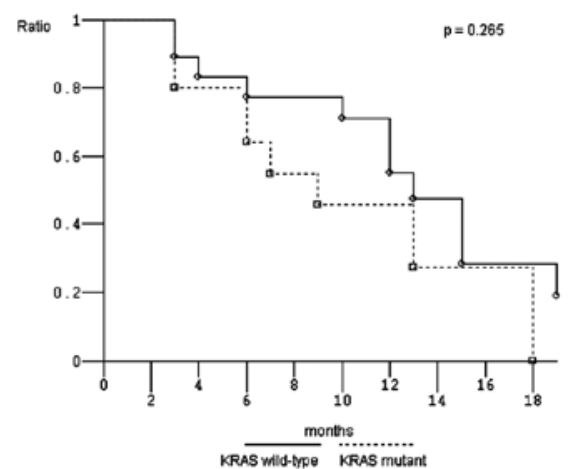

Figure 1. Kaplan-Meier survival estimate. (A) Overall treated patients; (B) L-L versus O/MM; (C) Overall population, KRAS wild-type versus KRAS mutant; (1) PFS; (2) OS. 
Table III. Overall activity and efficacy.

A, Activity, efficacy and effectiveness of first line regimens in unfit patients according to KRAS genotype

\begin{tabular}{|c|c|c|c|c|c|c|}
\hline & \multicolumn{2}{|c|}{$\begin{array}{c}\text { All treated } \\
\text { Intent-to-treat } \\
\text { Analysis }\end{array}$} & \multicolumn{2}{|c|}{$\begin{array}{c}K R A S \text { wild-type } \\
\text { Intent-to-treat } \\
\text { Analysis }\end{array}$} & \multicolumn{2}{|c|}{$\begin{array}{c}K R A S \text { mutant } \\
\text { Intent-to-treat } \\
\text { Analysis }\end{array}$} \\
\hline & No. & $\%$ & No. & $\%$ & No. & $\%$ \\
\hline Enrolled patients & 37 & 100 & 21 & 100 & 15 & 100 \\
\hline Evaluable patients & 27 & 70 & 14 & 67 & 12 & 80 \\
\hline Objective response & 10 & $37(\mathrm{CI} \pm 19)$ & 7 & $50(\mathrm{CI} \pm 27)$ & 3 & $25(\mathrm{CI} \pm 26)$ \\
\hline Partial response & 9 & 33 & 6 & 43 & 3 & 25 \\
\hline Complete response & 1 & 4 & 1 & 7 & - & - \\
\hline Stable disease & 9 & 33 & 4 & 29 & 5 & 42 \\
\hline Progressive disease & 8 & 30 & 3 & 21 & 4 & 33 \\
\hline Median PFS, months & 7 & & 8 & & 6 & \\
\hline Range & $1-13+$ & & $1+-13+$ & & $1-11$ & \\
\hline Progression events & 28 & 76 & 15 & 71 & 12 & 80 \\
\hline Median OS, months & 13 & & 13 & & 9 & \\
\hline Range & $1+-23+$ & & $1+-23+$ & & $3-18$ & \\
\hline Deaths & 22 & 59 & 11 & 52 & 10 & 67 \\
\hline Liver metastasectomies & 3 & & 3 & & - & \\
\hline No/overall pts & $3 / 37$ & 8 & $3 / 21$ & 14 & - & - \\
\hline No/patients with liver metastases & $3 / 26$ & 11.5 & $3 / 16$ & 19 & - & - \\
\hline No/patients with L-L metastases & $3 / 8$ & 37.5 & $3 / 7$ & 43 & - & - \\
\hline Pathologic complete responses & 1 & 33 & 1 & 33 & - & - \\
\hline
\end{tabular}

PFS, progression-free survival; OS, overall survival, L-L, liver-limited.

B, Activity, efficacy and effectiveness according to first line treatments

\begin{tabular}{|c|c|c|c|c|}
\hline & \multicolumn{4}{|c|}{ Intent-to-treat analysis } \\
\hline & \multicolumn{2}{|c|}{ Triplet regimen } & \multicolumn{2}{|c|}{ Doublet regimen } \\
\hline & No. & $\%$ & No. & $\%$ \\
\hline Enrolled patients & 18 & 100 & 15 & 100 \\
\hline Evaluable patients & 16 & 89 & 9 & 60 \\
\hline Objective response & 6 & $37.5(\mathrm{CI} \pm 24)$ & 4 & $44(\mathrm{CI} \pm 34)$ \\
\hline Partial response & 6 & 37.5 & 3 & 33 \\
\hline Complete response & - & - & 1 & 11 \\
\hline Stable disease & 5 & 31 & 3 & 33 \\
\hline Progressive disease & 5 & 31 & 2 & 22 \\
\hline Median PFS, months & 8 & & 8 & \\
\hline Range & $3-12$ & & $1-13+$ & \\
\hline Progression events & 14 & 78 & 9 & 60 \\
\hline Median OS, months & 12 & & 15 & \\
\hline Range & $3-23+$ & & $1+-23+$ & \\
\hline Deaths & 12 & 67 & 7 & 47 \\
\hline Liver metastasectomies & 1 & 6 & 1 & 11 \\
\hline Pathologic complete responses & 1 & 100 & - & - \\
\hline
\end{tabular}

PFS, progression-free survival; OS, overall survival. 
A1

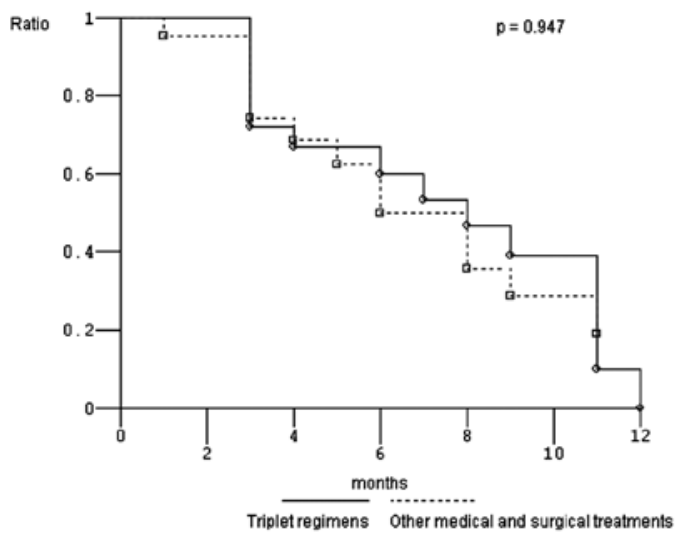

B1

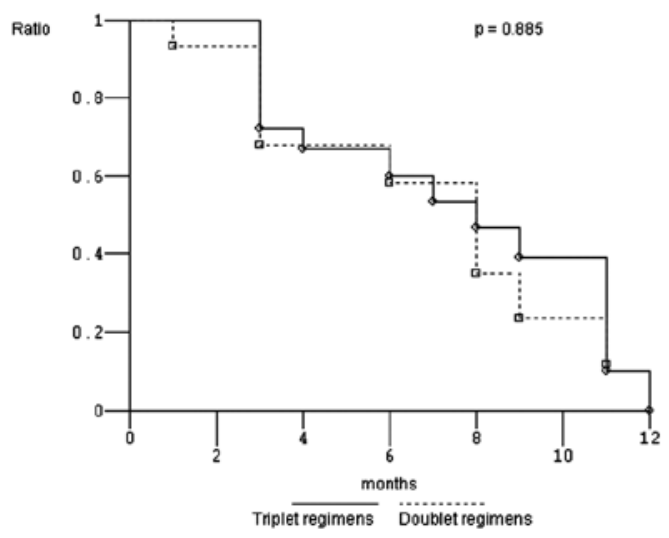

A2

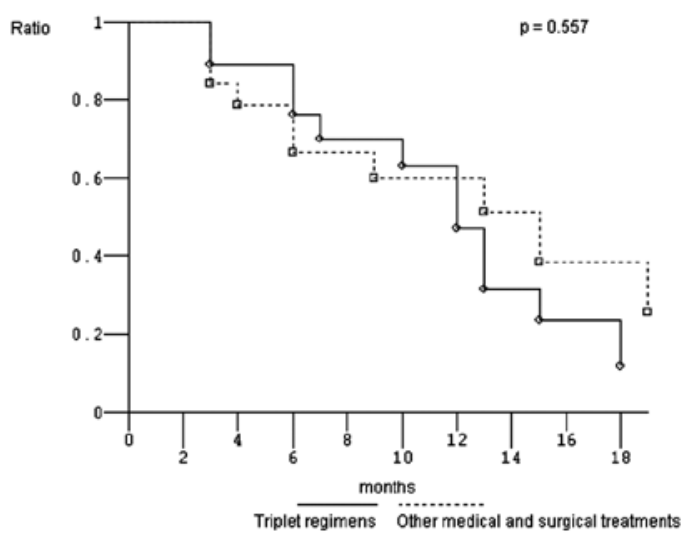

B2

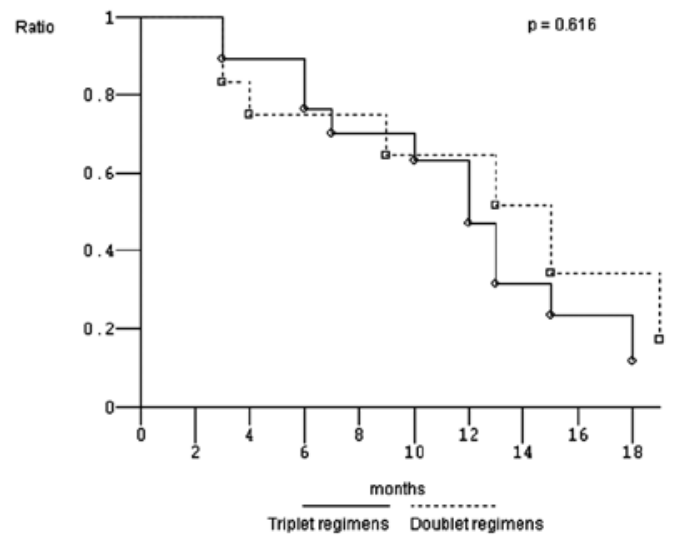

Figure 2. Kaplan-Meier survival estimate. (A) First line treatment, triplet regimens versus other medical and surgical treatments. (B) First line treatment, triplet regimens versus doublet regimens. (1) PFS; (2) OS.

8 months (3-12): 14 events occurred. Median OS was 12 months (3-23+ months): 12 events occurred. Secondary metastasectomy was performed in 1 patient (6\%). Among 15 patients treated with doublet regimens (Table IIIB), ORR was $44 \%$ $(\alpha 0.05, \mathrm{CI} \pm 34)$. We observed 3 partial responses (33\%); $1 \mathrm{CR}$ (11\%); 3 stable diseases (33\%); 2 progressive diseases (22\%). Median PFS was 8 months (1-13+): 9 events occurred. Median OS was 15 months (1+-23+ months): 7 events occurred. Among 4 patients treated with mono-regimens, median PFS was 5 months (3-6 months), median OS 6 months (3-13+ months). Among 3 patients who underwent surgery as first line treatment, median PFS was not reached (3+-19+ months); median OS not reached (3+-19+ months). PFS and OS were not significantly different in patients treated with triplet compared to other first line treatments $(\mathrm{p}=0.947$ and 0.557 , respectively), and to doublet regimens ( $\mathrm{p}=0.885$ and 0.616 , respectively) (Fig. 2).

Moreover, PFS and OS were not significantly different in non-elderly and young-elderly compared to old-elderly patients ( $\mathrm{p}=0.240$ and 0.750 , respectively), and in primary and intermediate CIRS stage compared to secondary stage patients ( $\mathrm{p}=0.494$ and 0.364 , respectively).

Prognostic relevance of KRAS genotype and c.35 G>A KRAS mutation. Among $14 K R A S$ wild-type patients evaluable for activity, ORR was $50 \%(\alpha 0.05, \mathrm{CI} \pm 27)$ (Table IIIA). We observed 7 objective responses: 6 partial (43\%) and 1 CR (7\%); 4 stable diseases (29\%); 3 progressive diseases (21\%). Disease control rate was $79 \%(\alpha 0.05, \mathrm{CI} \pm 22)$. Liver metastasectomies were performed in 3 patients (14\%), 3 out of 7 L-L (43\%). Median PFS was 8 months (1+-13+ months), 15 events occurred (71\%). Median OS was 13 months (1+-23+ months), 11 events occurred. Among 12 KRAS mutant patients evaluable for activity, ORR was $25 \%(\alpha 0.05, \mathrm{CI} \pm 26)$. We observed 3 partial responses (25\%); 5 stable diseases $(42 \%)$; 4 progressive diseases (33\%). Disease control rate was $67 \%(\alpha 0.05, \mathrm{CI} \pm 28)$. No liver metastasectomies were performed. Median PFS was 6 months (1-11 months), 12 events occurred (80\%). Median OS was 8 months (3-18 months), 10 events occurred. KRAS wild-type compared with mutant patients showed significantly different PFS (p=0.043), but not OS (Fig. 1C). KRAS c.35 G>A mutant patients showed significantly worse PFS and OS compared to wild-type ( $\mathrm{p}=0.000$, and 0.049 , respectively) (Fig. 3A and B), and to other mutant patients $(\mathrm{p}=0.020$ and 0.048 , respectively) (Fig. 3C and D). No different clinical outcomes were reported in other than c.35 G>A KRAS mutant compared to wild-type patients (Fig. 3E and F). PFS and OS were also significantly worse in c. $35 \mathrm{G}>\mathrm{A}$ KRAS mutant patients compared to other mutant plus wild-type patients $(\mathrm{p}=0.000$, and 0.021 , respectively) (Fig. 3G and $\mathrm{H}$ ). 
A

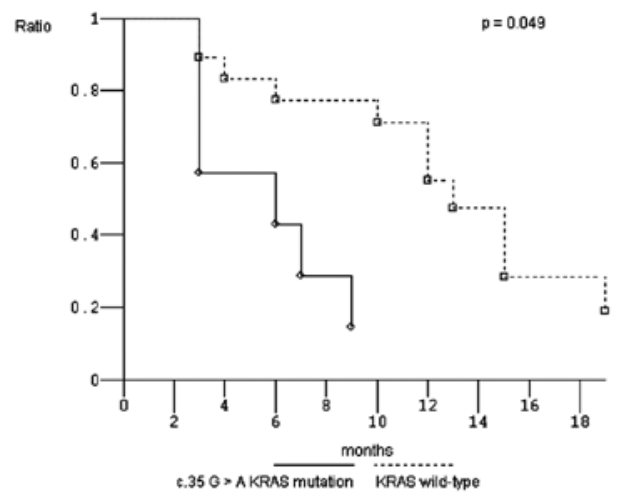

C

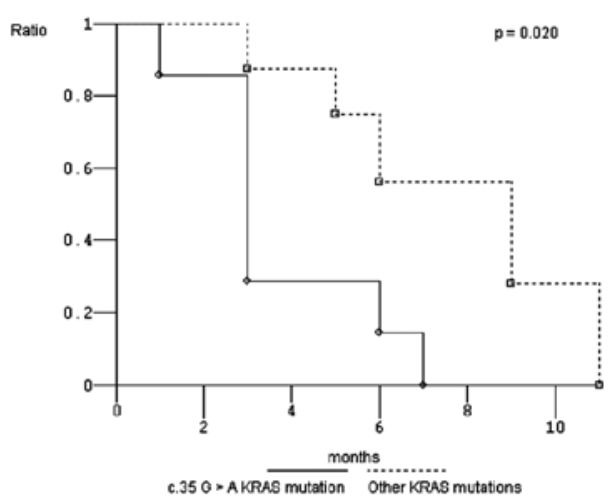

E

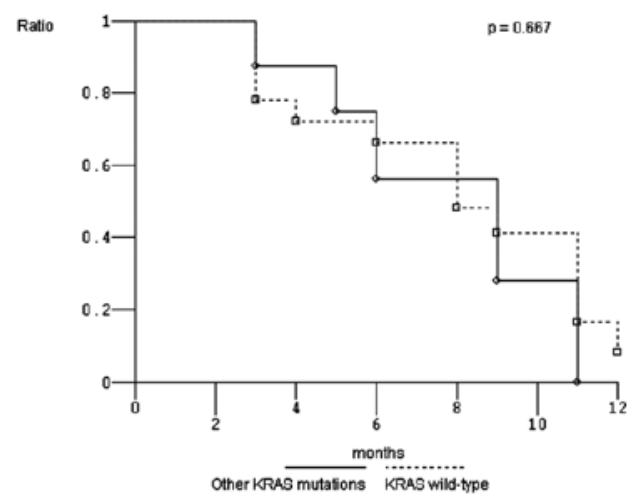

G

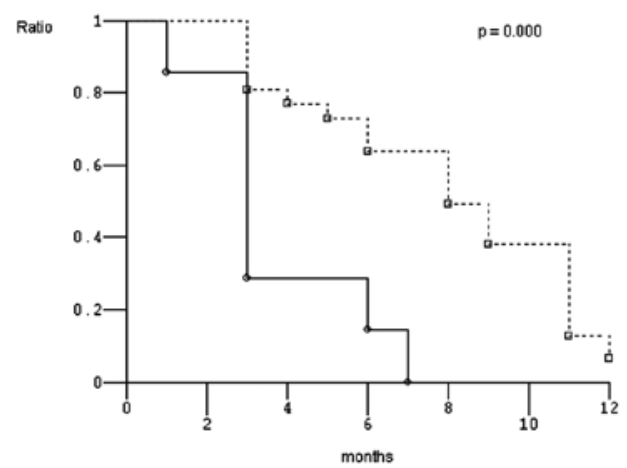

c.35 Q $\times$ A KRAS mutation Other KRAS mutations and KRAS Wild-trpe
B

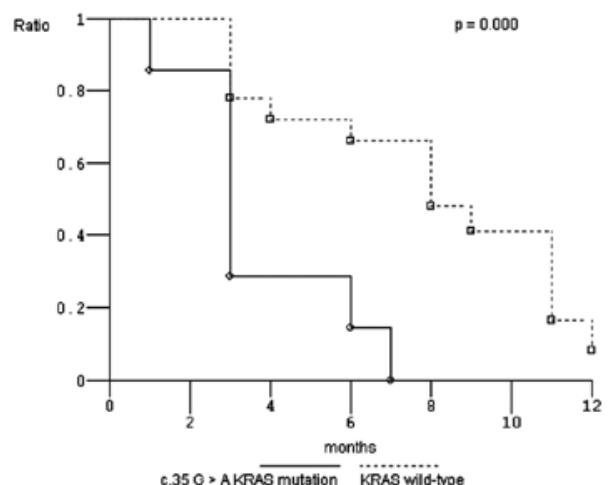

D

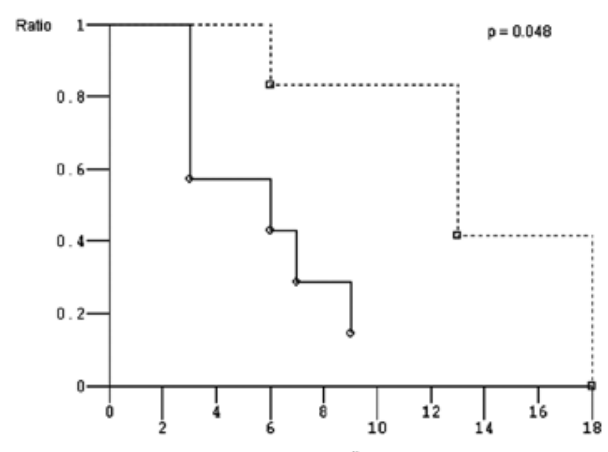

c.35 G > A KRAS mutation

F

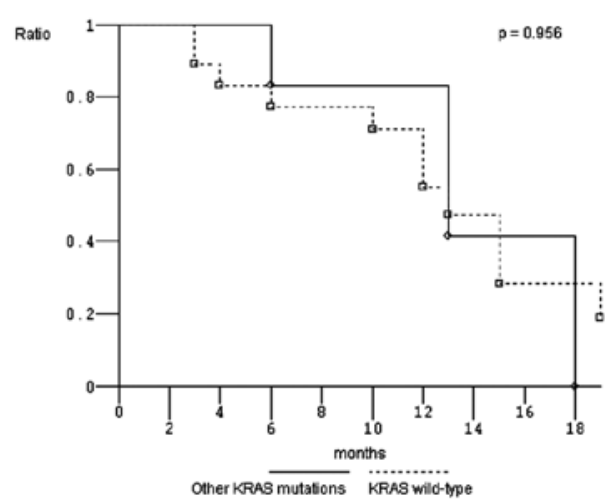

H

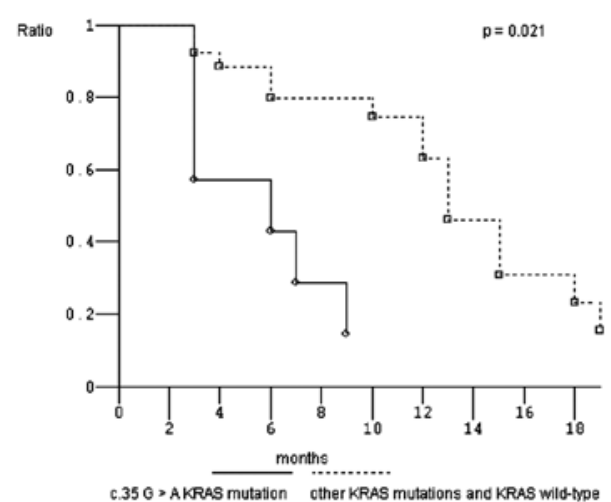

Figure 3. Kaplan-Meier survival estimate. (A) PFS c. 35 G>A KRAS mutant versus wild-type patients; (B) OS c.35 G>A KRAS mutant versus wild-type patients; (C) PFS c.35 G>A KRAS mutant versus other mutant patients; (D) OS c.35 G>A KRAS mutant versus other mutant patients; (E) PFS other KRAS mutant versus wild-type patients; (F) OS other KRAS mutant versus wild-type patients; (G) PFS c.35 G>A KRAS mutant versus other mutant plus wild-type patients; (H) OS c.35 G>A KRAS mutant versus other mutant plus wild-type patients. 


\section{Discussion}

Patients unfit for first line FIr-B/FOx intensive regimen, due to age ( $\geq 75$ years) and/or comorbidities, were prevalent $(56 \%)$, mostly elderly (76\%), particularly old-elderly patients (54\%), prevalently PS 1-2 (59\%), CIRS stage intermediate/secondary (89\%), O/MM disease (79\%). Most unfit MCRC patients were treated with triplet or doublet regimens (49 and 40\%, respectively), and some (19\%) did not reach the first evaluation of activity at 2-3 months.

Retrospective evaluations of doublets consisting of CPT-11 or OXP, associated to 5-FU or capecitabine in elderly patients eligible for clinical trials gained ORR $18-59.4 \%$, PFS 4.9-10.0 months and OS 8.5-20.7 months (11-16,20,30,31,46). BEV addition to 5-FU-based chemotherapy in elderly patients significantly increased PFS up to 9.2-9.3 months and OS up to 17.4-19.3 months $(22,23)$. Triplet chemotherapy or doublets plus BEV obtained ORR 34.9-45.9\%, PFS 7.9-9.3 months and OS 17.4-20.5 months (23-25). The present tailored approach, based on evaluation of elderly status and/or CIRS, prevalently addressing doublet and triplet regimens, reported ORR 37\%, PFS 7 months and OS 13 months. Selected medical treatment, triplet compared to doublet, did not significantly affected PFS and OS, nor advanced age, or CIRS stage. The FOCUS2 randomized trial evaluating first line reduced dose 5-FU or capecitabine and OXP in old-elderly and/or frail patients showed significantly improved ORR $35 \%$, with PFS 5.8 months (31). The meta-analysis evaluating the effect of PS on clinical outcome showed that PS 1 compared to PS 2 significantly affected prognosis, regardless of treatment, with ORR 43.8 vs $32 \%$, PFS 7.6 vs 4.9 months, OS 17.3 and 8.5 months, respectively (30). In the HORG-FOLFOXIRI trial, elderly compared to non-elderly patients treated with FOLFIRI or FOLFOXIRI showed no different clinical outcome; significantly lower PFS and OS were reported in patients with PS 2 (26,27).

Young-elderly patients eligible for FIr-B/FOx intensive regimen, prevalently characterised by PS $0(89 \%)$ and intermediate CIRS stage (93\%), reported ORR 79\%, PFS 11 months, OS 21 months, equivalent to overall patients (7,47). A complex decision-making process discriminating patients' fitness, and tailoring a personalized medical treatment, is challenging: patients unfit for FIr-B/FOx can be treated with a two-drug first line combination regimen (31), but showed worse clinical outcome. No increased morbidity, nor mortality was reported in unfit patients who underwent secondary liver metastasectomies, reported as significantly higher in elderly patients (8\%) (48).

Overall in MCRC patients treated with BEV added to CPT-11/5-FU, or with more intensive regimens (FIr-B/FOx, FOLFOXIRI/BEV), PFS and OS were not significantly different in KRAS wild-type and mutant $(4,6,8,37)$ as well as in young-elderly patients (47). Recently, KRAS genotype was reported as significantly affecting PFS and OS in patients treated with XelOx/BEV (49). We recently reported in MCRC patients treated with FIr-B/FOx, that the prevalent $K R A S$ c. $35 \mathrm{G}>\mathrm{A}$ (G12D) mutant genotype may significantly affect worse OS, compared to wild-type or other mutations (40). Present data reported for the first time that in patients unfit for FIr-B/FOx, KRAS wild-type compared to mutant patients showed a significantly different PFS, and not OS. Furthermore, KRAS c.35 G>A mutant genotype may affect significantly worse PFS and OS, compared to wild-type and/ or other mutant, confirming that KRAS genotype, particularly c. $35 \mathrm{G}>\mathrm{A}$ mutant, confers different biological aggressiveness (35), less effectively overcome by conventional triplet and doublet regimens. The prognostic relevance of KRAS genotype, particularly c. $35 \mathrm{G}>\mathrm{A}$ mutant $(4,40)$, and the predictive relevance of different medical treatments according to patients' fitness for intensive regimens, should be prospectively evaluated.

In conclusion, in MCRC patients unfit for first line intensive FIr-B/FOx regimen, tailored doublet and triplet medical treatments showed similar activity and efficacy, also according to age and comorbidities. KRAS genotype may indicate different PFS, and c. $35 \mathrm{G}>\mathrm{A} K R A S$ mutant a significantly worse PFS and OS, compared to wild-type and other mutations. Present findings warrant prospective trials comparing clinical outcome in unfit patients, according to KRAS genotype.

\section{Acknowledgements}

G.B. is a PhD student in Biotechnology, Department of Biotechnological and Applied Clinical Sciences, University of L'Aquila, funded by the University of L'Aquila, Italy.

\section{References}

1. Schmoll HJ, Van Cutsem E, Stein A, Valentini V, Glimelius B, Haustermans K, Nordlinger B, van de Velde CJ, Balmana J, Regula J, Nagtegaal ID, Beets-Tan RG, Arnold D, Ciardiello F, Hoff P, Kerr D, Köhne CH, Labianca R, Price T, Scheithauer W, Sobrero A, Tabernero J, Aderka D, Barroso S, Bodoky G, Douillard JY, El Ghazaly H, Gallardo J, Garin A, Glynne-Jones R, Jordan K, Meshcheryakov A, Papamichail D, Pfeiffer P, Souglakos I, Turhal S and Cervantes A: ESMO Consensus Guidelines for management of patients with colon and rectal cancer. A personalized approach to clinical decision making. Ann Oncol 23: 2479-2516, 2012.

2. Ficorella C, Bruera G, Cannita K, Porzio G, Lanfiuti Baldi P, Tinari N, Natoli $C$ and Ricevuto E: Triplet chemotherapy in patients with metastatic colorectal cancer: toward the best way to safely administer a highly active regimen in clinical practice. Clin Colorectal Cancer 11: 229-237, 2012.

3. Bruera G, Cannita K, Giuliante F, Lanfiuti Baldi P, Vicentini R, Marchetti P, Nuzzo G, Antonucci A, Ficorella C and Ricevuto E: Effectiveness of liver metastasectomies in patients with metastatic colorectal cancer treated with FIr-B/FOx triplet chemotherapy plus bevacizumab. Clin Colorectal Cancer 11: 119-126, 2012.

4. Bruera G, Cannita K, Di Giacomo D, Lamy A, Troncone G, Dal Mas A, Coletti G, Frébourg T, Sabourin JC, Tosi M, Ficorella $\mathrm{C}$ and Ricevuto E: Prognostic value of KRAS genotype in metastatic colorectal cancer (MCRC) patients treated with intensive triplet chemotherapy plus bevacizumab (FIr-B/FOx) according to extension of metastatic disease. BMC Med 10: 135, 2012.

5. Bruera G and Ricevuto E: Intensive chemotherapy of metastatic colorectal cancer: weighing between safety and clinical efficacy. Evaluation of Masi G, Loupakis F, Salvatore L, et al: Bevacizumab with FOLFOXIRI (irinotecan, oxaliplatin, fluorouracil, and folinate) as first-line treatment for metastatic colorectal cancer: a phase 2 trial. Expert Opin Biol Ther 11: 21-824, 2011.

6. Masi G, Vasile E, Loupakis F, Cupini S, Fornaro L, Baldi G, Salvatore L, Cremolini C, Stasi I, Brunetti I, Fabbri MA, Pugliesi M, Trenta P, Granetto C, Chiara S, Fioretto L, Allegrini G, Crinò L, Andreuccetti $M$ and Falcone A: Randomized trial of two induction chemotherapy regimens in metastatic colorectal cancer: an updated analysis. J Natl Cancer Inst 103: 21-30, 2011. 
7. Bruera G, Santomagoio A, Cannita K, Lanfiuti Baldi P, Tudini M, De Galitiis F, Mancini M, Marchetti P, Antonucci A, Ficorella C and Ricevuto E: 'Poker' association of weekly alternating 5-fluorouracil, irinotecan, bevacizumab and oxaliplatin (FIr-B/FOx) in first line treatment of metastatic colorectal cancer: a phase II study. BMC Cancer 10: 567, 2010.

8. Masi G, Loupakis F, Salvatore L, Fornaro L, Cremolini C, Cupini S, Ciarlo A, Del Monte F, Cortesi E, Amoroso D, Granetto C, Fontanini G, Sensi E, Lupi C, Andreuccetti M and Falcone A: Bevacizumab with FOLFOXIRI (irinotecan, oxaliplatin, fluorouracil, and folinate) as first-line treatment for metastatic colorectal cancer: a phase 2 trial. Lancet Oncol 11: $845-852,2010$

9. Garufi C, Torsello A, Tumolo S, Ettorre GM, Zeuli M, Campanella C, Vennarecci G, Mottolese M, Sperduti I and Cognetti F: Cetuximab plus chronomodulated irinotecan, 5-fluorouracil, leucovorin and oxaliplatin as neoadiuvant chemotherapy in colorectal liver metastases: POCHER trial. Br J Cancer 103: $1542-1547,2010$

10. Extermann M, Overcash J, Lyman GH, Parr J and Balducci L: Comorbidity and functional status are independent in older cancer patients. J Clin Oncol 16: 1582-1587, 1998

11. Folprecht G, Cunningham D, Ross P, Glimelius B, Di Costanzo F, Wils J, Scheithauer W, Rougier P, Aranda E, Hecker H and Kohne $\mathrm{CH}$ : Efficacy of 5-fluorouracil-based chemotherapy in elderly patients with metastatic colorectal cancer: a pooled analysis of clinical trials. Ann Oncol 15: 1330-1338, 2004.

12. Popescu RA, Norman A, Ross PJ, Parikh B and Cunningham D: Adjuvant or palliative chemotherapy for colorectal cancer in patients 70 years or older. J Clin Oncol 17: 2412-2418, 1999.

13. Chiara S, Nobile MT, Vincenti M, Lionetto R, Gozza A, Barzacchi MC, Sanguineti O, Repetto L and Rosso R: Advanced colorectal cancer in the elderly: results of consecutive trials with 5-fluorouracil-based chemotherapy. Cancer Chemother Pharmacol 42: 336-340, 1998

14. Folprecht G, Seymour MT, Saltz L, Douillard JY, Hecker H, Stephens RJ, Maughan TS, Van Cutsem E, Rougier P, Mitry E, Schubert U and Kohne CH: Irinotecan/fluorouracil combination in first-line therapy of older and younger patients with metastatic colorectal cancer: combined analysis of 2,691 patients in randomized controlled trials. J Clin Oncol 26: 1443-1451, 2008.

15. Mitry E, Douillard JY, Van Cutsem E, Cunningham D, Magherini E, Mery-Mignard D, Awad L and Rouigier P: Predictive factors of survival in patients with advanced colorectal cancer: an individual data analysis of 602 patients included in irinotecan phase III trials. Ann Oncol 15: 1013-1017, 2004

16. Goldberg RM, Tabah-Fisch I, Bleiberg H, de Gramont A, Tournigand C, Andre T, Rothenberg ML, Green E and Sargent DJ: Pooled analysis of safety and efficacy of oxaliplatin plus fluorouracil/leucovorin administered bimonthly in elderly patients with colorectal cancer. J Clin Oncol 24: 4085-4091, 2006.

17. Pallis AG, Papamichael D, Audisio R, Peeters M, Folprecht G, Lacombe D and Van Cutsem E: EORTC Elderly Task Force experts' opinion for the treatment of colon cancer in older patients. Cancer Treat Rev 36: 83-90, 2010.

18. Papamichael D, Audisio R, Horiot JC, Glimelius B, Sastre J, Mitry E, Van Cutsem E, Gosney M, Kohne CH and Aapro M: Treatment of the elderly colorectal cancer patient: SIOG expert recommendations. Ann Oncol 20: 5-16, 2009.

19. Audisio RA and Papamichael D: Treatment of colorectal cancer in older patients. Nat Rev Gastroenterol Hepatol 9: 716-725, 2012.

20. Figer A, Perez-Staub N, Carola E, Tournigand C, Lledo G, Flesch M, Barcelo R, Cervantes A, André T, Colin P, Louvet C and de Gramont A: FOLFOX in patients aged between 76 and 80 years with metastatic colorectal cancer an exploratory cohort of the OPTIMOX1 study. Cancer 110: 2666-2671, 2007.

21. Hurwitz HI, Fehrenbacher L, Novotny WF, Cartwright T, Hainsworth J, Meropol NJ, Heim W, Berlin J, Baron A, Griffing S, Holmgren E, Ferrara N, Fyfe G, Rogers B, Ross M and Kabbinavar FF: Bevacizumab plus irinotecan, fluorouracil, and leucovorin for metastatic colorectal cancer. N Engl J Med 350: 2335-2342, 2004

22. Kabbinavar FF, Hurwitz HI, Yi J, Sarkar S and Rosen O: Addition of bevacizumab to fluorouracil-based first-line treatment of metastatic colorectal cancer: pooled analysis of cohorts of older patients from two randomized clinical trials. J Clin Oncol 27: 199-205, 2008

23. Cassidy J, Saltz LB, Giantonio BJ, Kabbinavar FF, Hurwitz HI and Rohr UP: Effect of bevacizumab in older patients with metastatic colorectal cancer: pooled analysis of four randomized studies. J Cancer Res Clin Oncol 136: 737-743, 2010.
24. Kozloff MF, Berlin J, Flynn PJ, Kabbinavar F, Ashby M, Dong W, Sing AP, Grothey A, for the BRiTE Investigators: Clinical outcomes in elderly patients with metastatic colorectal cancer receiving bevacizumab and chemotherapy: results from the BRiTE observational cohort study. Oncology 78: 329-339, 2010.

25. Van Cutsem E, Rivera F, Berry S, Kretzschmar A, Michael M, Di Bartolomeo M, Mazier MA, Canon JL, Georgoulias V, Peeters M, Bridgewater $\mathrm{J}$ and Cunningham $\mathrm{D}$, on behalf of the First BEAT investigators: Safety and efficacy of first-line bevacizumab with FOLFOX, XELOX, FOLFIRI and fluoropyrimidines in metastatic colorectal cancer: the BEAT study. Ann Oncol 20: 1842-1847, 2009

26. Souglakos J, Andrulakis N, Syrigos K, Polyzos A, Ziras N, Athanasiadis A, Kalolyris S, Tsousis S, Kouroussis $\mathrm{CH}$ Vamvakas L, Kalykaki A, Samonis G, Mavroudis D and Georgoulias V: FOLFOXIRI (folin acid, 5-fluorouracil, oxaliplatin and irinotecan) vs FOLFIRI (folin acid, 5-fluorouracil and irinotecan) as first-line treatment in metestatic colorectal cancer (MCC): a multicentre randomised phase III trial from the Hellenic Oncology Research Group (HORG). Br J Cancer 94: 798-805, 2006

27. Vamvakas L, Athanasiadis A, Karampeazis A, Kakolyris S, Polyzos A, Kouroussis C, Ziras N, Kalbakis K, Georgoulias V and Souglakos J: Clinical outcome of elderly patients with metastatic colorectal cancer treated with FOLFOXIRI versus FOLFIRI: Subgroup analysis of a randomized phase III trial from the Hellenic Oncology Research Group (HORG). Crit Rev Oncol Hematol 76: 61-70, 2010.

28. Meyerhardt JA, Jackson McCleary N, Niedzwiecki D, Hollis D, Venook A, Mayer R and Goldberg R: Impact of age and comorbidities on treatment effect, tolerance, and toxicity in metastatic colorectal cancer (mCRC) patients treated on CALGB 80203. J Clin Oncol 27 (Suppl 15): 4038, 2009.

29. Douillard J, Siena S, Cassidy J, Tabernero J, Burkes R, Barugel M, Humblet Y, Bodoky G, Cunningham D, Jassem J, Rivera F, Kocàkova I, Ruff P, Blasinska-Morawiec M, Smakal M, Canon JL, Rother M, Oliner KS, Wolf $M$ and Gansert J: Randomized, phase III trial of Panitumumab with infusional fluorouracil, leicovorin, and oxaliplatin (FOLFOX4) versus FOLFOX4 alone as first-line treatment in patients with previously untreated metastatic colorectal cancer: the PRIME trial. J Clin Oncol 28: 4697-4705, 2010.

30. Sargent DJ, Kohne CH, Kelly Sanoff H, Bot BM, Seymour MT, de Gramont A, Porschen R, Saltz LB, Rougier P, Tournigand C, Douillard JY, Stephens RJ, Grothey A and Goldberg RM: Pooled safety and efficacy analysis examining the effect of performance status on outcomes in nine first-line treatment trials using individual data from patients with metastatic colorectal cancer. J Clin Oncol 27: 1948-1955, 2009.

31. Seymour MT, Thompson LC, Wasan HS, Middleton G, Brewster AE, Shepherd SF, O'Mahony MS, Maughan TS, Parmar $M$ and Langley RE, on behalf of the FOCUS2 Investigators, and the National Cancer Research Institute Colorectal Cancer Clinical Studies Group: Chemotherapy options in elderly and frail patients with metastatic colorectal cancer (MRC FOCUS2): an open-label, randomised factorial trial. Lancet 377: 1749-1759, 2011.

32. Andreyev HJ, Norman AR, Cunningham D, Oates J, Dix BR, Iacopetta BJ, Young Y, Walsh T, Ward R, Hawkins N, Beranek M, Jandik P, Benamouzig R, Jullian E, Laurent-Puig P, Olschwang S, Muller O, Hoffmann I, Rabes HM, Zietz C, Troungos C, Valavanis C, Yuen ST, Ho JWC, Croke CT, O'Donoghue DP, Giaretti W, Rapallo A, Russo A, Bazan V, et al: Kirsten ras mutations in patients with colorectal cancer: the 'RASCAL II' study. Br J Cancer 85: 692-696, 2001.

33. Normanno N, Tejpar S, Morbillo F, De Luca A, Van Cutsem E and Ciardiello F: Implication of KRAS status and EGFRtargeted therapies in metastatic CRC. Nat Rev Clin Oncol 6: 519-527, 2009.

34. De Roock W, Claes B, Bernasconi D, De Schutter J, Biesmans B, Fountzilas G, Kalogeras KT, Kotoula V, Papamichael D, Laurent-Puig P, Penault-Llorca F, Rougier P, Vincenzi B, Santini D, Tonini G, Cappuzzo F, Frattini M, Molinari F, Saletti P, De Dosso S, Martini M, Bardelli A, Siena S, SartoreBianchi A, Tabernero J, Macarulla T, Di Fiore F, Gangloff AO, Ciardiello F, Pfeiffer P, et al: Effects of KRAS, BRAF, NRAS, and PIK3CA mutations on the efficacy of cetuximab plus chemotherapy in chemotherapy-refractory metastatic colorectal cancer: a retrospective consortium analysis. Lancet Oncol 11: 753-762, 2010 
35. Guerrero S, Casanova I, Farrè L, Mazo A, Capellà G and Mangues R: K-ras codon 12 mutation induces higher level of resistance to apoptosis and predisposition to anchorageindependent growth than codon 13 mutation or proto-oncogene overexpression. Cancer Res 60: 6750-6756, 2000.

36. Ince WL, Jubb AM, Holden SN, Holmgren EB, Tobin P, Sridhar M, Hurwitz HI, Kabbinavar F, Novotny WF, Hillan KJ and Koeppen H: Association of K-ras, B-raf, and p53 status with the treatment effect of bevacizumab. J Natl Cancer Inst 97: 981-989, 2005.

37. Hurwitz HI, Yi J, Ince W, Novotny WF and Rosen O: The clinical benefit of bevacizumab in metastatic colorectal cancer is independent of K-ras mutation status: analysis of a phase III study of bevacizumab with chemotherapy in previously untreated metastatic colorectal cancer. Oncologist 14: 22-28, 2009.

38. Van Cutsem E, Köhne CH, Làng I, Folprecht G, Nowacki MP, Cascinu S, Shchepotin I, Maurel J, Cunningham D, Tejpar S, Schlichting M, Zubel A, Celik I, Rougier P and Ciardiello F: Cetuximab plus irinotecan, fluorouracil, and leucovorin as first-line treatment for metastatic colorectal cancer: updated analysis of overall survival according to tumor KRAS and BRAF mutation status. J Clin Oncol 29: 2011-2019, 2011.

39. Bokemeyer C, Bondarenko I, Hartmann JT, de Braud F, Schuch G, Zubel A, Celik I, Schlichting M and Koralewski P: Efficacy according to biomarker status of cetuximab plus FOLFOX-4 as first-line treatment for metastatic colorectal cancer: the OPUS study. Ann Oncol 22: 1535-1546, 2011.

40. Bruera G, Cannita K, Di Giacomo D, Lamy A, Frébourg T, Sabourin JC, Tosi M, Alesse E, Ficorella C and Ricevuto E: Worse prognosis of KRAS c.35 G>A mutant metastatic colorectal cancer (MCRC) patients treated with intensive triplet chemotherapy plus bevacizumab (FIr-B/FOx). BMC Med 11: 59, 2013.

41. Morelli MF, Santomaggio A, Ricevuto E, Cannita K, De Galitiis F, Tudini M, Bruera G, Mancini M, Pelliccione M, Calista F, Guglielmi F, Martella F, Lanfiuti Baldi P, Porzio G, Russo A, Gebbia N, Iacobelli S, Marchetti P and Ficorella C, on behalf of CINBO (Consorzio Interuniversitario Nazionale per la Bio-Oncologia): Triplet schedule of weekly 5-fluorouraci and alternating irinotecan or oxaliplatin in advanced colorecta cancer: a dose-finding and phase II study. Oncol Rep 23: 1635-1640, 2010

42. Ficorella C, Ricevuto E, Morelli MF, Morese R, Cannita K, Cianci G, Di Rocco ZC, De Galitiis F, De Tursi M, Tinari N, Iacobelli S and Marchetti P:Increased tolerability of bimonthly 12-hour timed flat infusion 5-fluorouracil/irinotecan regimen in advanced colorectal cancer: a dose-finding study. Oncol Rep 15 $1345-1350,2006$.
43. Therasse P, Arbuck SG, Eisenhauer EA, Wanders J, Kaplan RS, Rubinstein L, Verweij J, Glabbeke MV, van Oosterom AT, Christian MC and Gwyther SG: New guidelines to evaluate the response to treatment in solid tumors: European Organization for Research and Treatment of Cancer, National Cancer Institute of the United States, National Cancer Institute of Canada. J Natl Cancer Inst 92: 205-216, 2000.

44. Kaplan EL and Meier P: Nonparametric estimation of incomplete observations. J Am Statist Assoc 53: 457-481, 1958.

45. Peto R and Peto J: Asymptomatically efficient rank invariant test procedures. J R Stat Soc A 135: 185-207, 1972.

46. Tebbutt NC, Wilson K, Gebski VJ, Cummins MM, Zannino D, van Hazel GA, Robinson B, Broad A, Ganju V, Ackland SP, Forgeson G, Cunningham D, Saunders MP, Stockler MR, Chua Y, Zalcberg JR, Simes RJ and Price TJ: Capecitabine, bevacizumab, and mitomycin in first-line treatment of metastatic colorectal cancer: results of the Australasian Gastrointestinal Trials Group randomized phase III MAX study. J Clin Oncol 28: 3191-3198, 2010.

47. Bruera G, Cannita K, Giordano AV, Vicentini R, Ficorella C and Ricevuto E: Effectiveness and safety of intensive triplet chemotherapy plus bevacizumab, FIr-B/FOx, in young-elderly Metastatic Colorectal Cancer (MCRC) patients. BioMed Res Int 2013: 143273, 2013.

48. Figueras J, Ramos E, López-Ben S, Torras J, Albiol M, Llado L, González HD and Rafecas A: Surgical treatment of liver metastases from colorectal carcinoma in elderly patients. When is it worthwhile? Clin Transl Oncol 9: 392-400, 2007.

49. Diaz-Rubio E, Gomez-Espana A, Massuti B, Sastre J, Reboredo M, Manzano JL, Rivera F, Safont Mj, Montagut C, lez EG, Benavides M, Marcuello E, Cervantes A, de Prado PM, Fernandez-Martos C, Arrivi A, Bando I and Aranda E on behalf of the Spanish Cooperative Group for the Treatment of Digestive Tumors (TTD): Role of Kras status in patients with metastatic colorectal cancer receiving first-line chemotherapy plus bevacizumab: a TTD group cooperative study. PLoS One 7: e47345, 2012. 